\title{
Uso de Hormônio de Crescimento na Síndrome de Prader-Willi
}

\begin{abstract}
RESUMO
A síndrome de Prader-Willi (PWS), com prevalência de 60:1.000.000, é o resultado da perda de parte do cromossomo 15 paterno, em razão da deleção em $56 \%$ dos casos, dissomia uniparental materna em $24 \%$ dos casos, ou por causa da metilação, fenômeno epigenético, em $18 \%$ dos casos. O quadro clínico inicia-se com profunda hipotonia que, especialmente no primeiro ano de vida, torna difícil a alimentação da criança. Conforme melhora a hipotonia, nos primeiros dois anos, por volta do quarto ano de vida, um apetite insaciável advém, o que leva tais crianças à obesidade extrema, com hipoventilação alveolar que põe em risco sua sobrevivência. Dessa forma, paradoxalmente, a PWS ameaça a vida dos pacientes, em um primeiro momento, por inanição e, em uma fase posterior, pelo excesso de peso. $\mathrm{O}$ uso de hormônio de crescimento (hrGH) nessas crianças tem por objetivo primário a mudança da composição corpórea e a melhora da atividade física e da qualidade de vida. Por outro lado, muitos pacientes com PWS são, de fato, deficientes em GH, ocorrendo melhora no padrão de crescimento com o tratamento. Tem-se de ser cuidadoso, no entanto, ao iniciar o tratamento com hrGH, com zelosa avaliação da apnéia do sono (polissonografia) e da permeabilidade das vias aéreas, tendo em vista que o tratamento com hrGH pode piorar o padrão respiratório em alguns pacientes. (Arq Bras Endocrinol Metab 2008; 52/5:833-838)
\end{abstract}

Descritores: Síndrome de Prader-Willi; Apnéia do sono; Hipotonia; Baixa estatura; Hipogonadismo; Hormônio de crescimento

\begin{abstract}
Growth Hormone usage in Prader-Willi Syndrome.

Prader-Willi syndrome (PWS), with a prevalence of 60:1.000.000, results from the loss of paternal chromosome 15 , being $56 \%$ due to deletion, $24 \%$ due to uniparental maternal disomy, and $18 \%$ from methylation, an epigenetic phenomenon. The clinical picture begins with extreme muscular hypotonia, which makes it difficult to feed the child in the first year. As the hypotonia improves, usually in the first two years, around the $4^{\text {th }}$ year of life, an insatiable appetite leads these children to an extreme obesity, with alveolar hypoventilation which endangers their lives. So, paradoxically, PWS threatens the lives of the patients, through inanition in a first phase and, afterwards, through excessive weight gain. The use of growth hormone $(\mathrm{hrGH})$ in these children has a primary goal to change the body composition and improve the physical activity and the quality of life. On the other hand, many PWS patients are indeed GH deficient, and an improvement in the height SDS occurs with treatment. We have to be careful, however. When starting a PWS treatment with a patient on $\mathrm{hrGH}$, a careful evaluation of sleep apnoea (polysomnography) as well as a careful examination of the airways is extremely mandatory, since the treatment may compromise the respiratory pattern of some patients. (Arq Bras Endocrinol Metab 2008; 52/5:833-838)
\end{abstract}

Keywords: Prader-Willi syndrome; Sleep apnoea; Hypotonia; Short stature; Hypogonadism; Growth hormone revisão

Durval Damiani

Unidade de Endocrinologia Pediátrica do Instituto da

Criança do Hospital das Clínicas da Faculdade de Medicina da Universidade de São Paulo (HC-FMUSP), SP, Brasil. 


\section{INTRODUÇÃO}

A síndrome de Prader-Willi (PWS) é um distúrbio neurogenético, descrito em 1956, que decorre da deleção da porção proximal do braço longo do cromossomo 15 paterno (15 q11-13) ou, mais raramente, de translocações, dissomia materna do cromossomo 15 ou anormalidades do imprinting cromossômico $(1,2)$. Apresenta incidência de cerca de 1:15.000 nascidos vivos e prevalência de 60:1.000.000 (3). Em um grande estudo italiano, incluindo 425 pacientes (209 do sexo masculino e 216 do sexo feminino), 238 pacientes (56\%) apresentavam deleção do cromossomo 15, 104 (24\%) dissomia uniparental materna do cromossomo 15 , quatro $(0,9 \%)$ apresentavam translocação afetando o cromossomo 15 e 79 (18\%) mostravam teste de metilação positivo (4). É a causa genética mais comum de obesidade mórbida na espécie humana. Até o momento, dois genes têm sido envolvidos na síndrome: o gene da necdina e o gene do polipeptídeo $\mathrm{N}$ da ribonucleoproteína.

Do ponto de vista clínico, a PWS apresenta aspecto até certo ponto paradoxal: a criança nasce apresentando grave hipotonia muscular, a tal ponto que até a alimentação torna-se difícil e não comumente, tais crianças devem ser alimentadas por sonda para não morrerem por inanição. Por outro lado, a partir do quarto ao sexto anos de vida, inicia-se apetite voraz e com progressivo ganho de peso, visto que o excesso alimentar novamente coloca em risco a vida do paciente, pois grande parte deles vai a óbito por hipoventilação alveolar, decorrente de extrema obesidade. Além dessas características, baixa estatura, baixa densidade mineral óssea, distúrbios do sono e alterações de comportamento, com "crises temperamentais" são encontrados, compondo um quadro clínico que se torna bastante característico e conduz ao diagnóstico (5). Vale ressaltar que apesar de a insuficiência hipotalâmica, incluindo o hipogonadismo hipogonadotrófico, ser a forma de apresentação mais comum, há poucos casos relatados de puberdade precoce central na PWS (6).

A deficiência mental na PWS costuma ser leve a moderada, com dificuldade de aprendizado em fase escolar. As alterações comportamentais e sociais podem estar presentes, especialmente a compulsão alimentar. São crianças e adolescentes "insaciáveis", o que conduz à séria obesidade se medidas restritivas alimentares não forem tomadas e mantidas.

A avaliação diagnóstica deve ser complementada pelo estudo do DNA. A Tabela 1 indica as situações clínicas em que se impõe a avaliação por biologia molecular.
Tabela 1. Critérios propostos para avaliação de pacientes suspeitos de serem portadores da PWS com estudo de DNA, de acordo com a idade (7).

\begin{tabular}{|ll}
\hline Age at assessment & Features sufficient to prompt \\
BNA testing
\end{tabular}

\section{ASPECTOS METABÓLICOS DA SÍNDROME DE PRADER-WILLI}

Do ponto de vista metabólico, chama a atenção que, apesar de o grau de obesidade, tais crianças não apresentam grande prevalência de resistência à insulina ou diabetes melito tipo 2 , talvez pelo fato de terem pouca gordura visceral. Em um estudo com 75 pacientes com PWS comparados a 395 pacientes obesos, Krochik e cols. observaram que, com índices de massa corporal comparáveis nos dois grupos $(30,08 \pm 9,7$ versus $30,5 \pm$ 4,8 no grupo de PWS e nos obesos, respectivamente), a presença de acantosis nigricans era de $29,3 \%$ versus $72 \%$, a pressão sistólica era mais baixa no grupo de PWS (104 \pm 16 versus $118 \pm 18$ ), a pressão diastólica era mais baixa $(63 \pm 13$ versus $75 \pm 13,6)$ e a insulinemia basal era mais baixa no grupo de PWS ( $12 \pm 8,2$ versus 22,3 \pm 25 ), sem diferenças nas glicemias de jejum e na inci- 
dência de diabetes melito tipo 2 (nenhum caso no grupo de PWS versus $1,5 \%$ no grupo obeso). A resistência à insulina, avaliada pelo índice HOMA-IR, mostrou-se significativamente mais baixa no grupo de PWS $(2,47 \pm$ $1,6$ versus $4,18 \pm 5,5)(8)$.

Quando se avalia a adiponectina, substância produzida pelo tecido adiposo e que tem ações antiaterogênicas, antiinflamatórias e antidiabetogênicas, verifica-se que, em crianças com PWS os níveis são elevados, em oposição ao que ocorre no obeso exógeno, apesar de tais crianças com a PWS terem maior porcentagem de gordura corpórea. Os níveis de adiponectina correlacionam-se inversamente ao de triglicerídeos. No momento em que essas crianças são tratadas com hormônio de crescimento, ocorre aumento adicional das concentrações de adiponectina (9).

É interessante observar que certas características metabólicas da PWS lembram as deficiências de hormônio de crescimento $(\mathrm{GH})$, como redução de massa magra, redução da concentração de fator de crescimento insulina-símile (IGF-1) e crescimento estatural reduzido (10). Quando foi avaliada a produção de $\mathrm{GH}$ nessas crianças, verificou-se que grande parte delas realmente apresenta deficiência de produção, talvez refletindo problema hipotalâmico, relacionado aos distúrbios alimentares e ao hipogonadismo hipogonadotrófico (11). O percentil 50 para estatura na PWS equivale ao percentil 5 da curva de crescimento de crianças normais na faixa etária de 12 a 14 anos. Além disso, a altura média para homens com PWS é de $155 \mathrm{~cm}$ e a de mulheres, $148 \mathrm{~cm}$. $(12,13)$. Quanto aos testes de estímulo, o pico de secreção de GH após clonidina em nossos pacientes foi de $0,8 \pm 0,8 \mathrm{ng} /$ $\mathrm{mL}$ (faixa de 0,2 a $1,8 \mathrm{ng} / \mathrm{mL}$ ) e o pico de $\mathrm{GH}$ após hipoglicemia insulínica foi de $2,9 \pm 2,6 \mathrm{ng} / \mathrm{mL}$ (faixa de $0,3$ a $0,6 \mathrm{ng} / \mathrm{mL})(5)$.

Quando o paciente com PWS foi comparado com o obeso exógeno, observa-se que a produção de IGF-1 neste último é mais elevada do que no paciente com a PWS, possivelmente decorrente da produção pelo adipócito. Apesar de abundante tecido adiposo, na PWS não há estímulo adequado à produção de IGF-1 (14).

Um outro aspecto interessante é que na PWS as concentrações de ghrelina estão elevadas, ao contrário do que ocorre com o obeso exógeno. A ghrelina, produzida no fundo gástrico, indica que o estômago está vazio, promovendo a sensação de fome. A ghrelina reduz-se após a ingestão de carboidratos ou de lípides, mas não de proteínas. Por outro lado, a ghrelina atua no receptor dos secretagogos de hormônio de cresci- mento e libera GH que, por retroinibição, impede a ghrelina. $\mathrm{O}$ nervo vago eferente contribui ao aumento de secreção de ghrelina induzida pelo jejum. Tais achados implicam o circuito vagal entre o sistema nervoso central e o estômago como crucial na regulação da concentração de ghrelina.

Além disso, o índice de massa corpórea modula os níveis de ghrelina plasmática. Em um indivíduo magro e na anorexia nervosa, a ghrelina está aumentada, enquanto na obesidade, com exceção à PWS, os níveis de ghrelina estão reduzidos e a redução induzida pela alimentação e pelo sono está comprometida. Há duas formas de ghrelina: n-octanoil e des-acil. O jejum aumenta ambos os tipos. A hiperfagia e a obesidade reduzem a des-acil ghrelina, mas não a n-octanoil. É possível que na PWS ocorra disfunção nessa relação entre os dois tipos de ghrelina, o que resultaria concentração reduzida, opondo-se ao que ocorre no obeso exógeno (15).

\section{AÇÕES METABÓLICAS DO HORMÔNIO DE CRESCIMENTO}

O hormônio de crescimento apresenta nome impróprio: em primeiro lugar, não é o único hormônio responsável pelo crescimento, já que o hormônio tireoidiano, a insulina e o cortisol, entre outros, também participam no processo de crescimento. Por outro lado, dizer que este polipeptídeo de 191 aminoácidos (na sua forma mais abundante, de $22 \mathrm{kDa}$ ) é só "de crescimento" é injustiça, já que os aspectos metabólicos deste composto não podem ser esquecidos.

Entre as ações metabólicas do GH, a alteração da composição corpórea, com aumento de massa magra e redução de massa gorda é a razão mais importante para seu uso tão generalizado e aceito na PWS. Seus efeitos no perfil lipídico implicam redução de colesterol total, LDL e triglicérides, fatores importantes na redução do risco cardiometabólico. Lanes e cols., ao comparar pacientes deficientes em GH tratados com deficientes em GH não-tratados e controles, mostram que há significativa redução de colesterol nos pacientes tratados, trazendo os níveis a valores semelhantes aos controles (154,9 $\mathrm{mg} / \mathrm{dL}$ em pacientes tratados, $190,4 \mathrm{mg} / \mathrm{dL}$ em nãotratados e $155,1 \mathrm{em}$ controles). O LDL colesterol em pacientes não-tratados apresenta média de $123,9 \mathrm{mg} /$ dL comparado a $95,9 \mathrm{mg} / \mathrm{dL}$ nos não-tratados e a 100,8 $\mathrm{mg} / \mathrm{dL}$ nos controles. Os triglicérides em não-tratados têm média de $88,3 \mathrm{mg} / \mathrm{dL}$ comparado a 74,6 e a 76,2 $\mathrm{mg} / \mathrm{dL}$ nos não-tratados e nos controles, respectiva- 
mente. Em relação aos níveis de HDL colesterol nesses três grupos não foram notadas diferenças. Outro aspecto interessante é que a adiponectina, uma adipocitocina antiaterogênica, antidiabetogênica e antiinflamatória, está reduzida em pacientes deficientes em $\mathrm{GH}$ não-tratados $(12,7 \mathrm{mcg} / \mathrm{mL})$, comparada aos tratados $(16,5 \mathrm{mcg} /$ $\mathrm{mL})$ e aos controles $(16,2 \mathrm{mcg} / \mathrm{mL})(16)$.

Em um estudo de 40 adolescentes, entre 16 e 21 anos de idade, avaliou-se a evolução de parâmetros metabólicos após cessar o uso de hormônio de crescimento recombinante humano (hrGH). Os pacientes foram retestados e 21 apresentavam deficiência grave de GH, enquanto 19 eram suficientes em GH. Em um acompanhamento de dois anos, verificou-se que, nos deficientes sem tratamento, o LDL colesterol se eleva, o HDL colesterol reduz-se e a massa gordurosa truncal é aumentada, em oposição aos controles normais e aos pacientes que receberam hrGH, mas que se mostram suficientes na produção de GH no momento (17).

Os efeitos cardiovasculares do $\mathrm{GH}$ foram avaliados por Salerno e cols. Os resultados apontam que em crianças deficientes em GH (13 pacientes estudados), houve redução da massa cardíaca, comparadas a 17 crianças com deficiência parcial de GH e com controles normais. O índice de massa ventricular esquerda era diminuído mesmo em pacientes com deficiência parcial de GH. Após um a dois anos de tratamento com hrGH, todos os índices se normalizaram (18).

Os adultos com deficiência de GH apresentam aumento de risco cardiovascular, representado pelo perfil lipídico desfavorável, aumento de gordura corpórea, aterosclerose prematura, reduzida atividade fibrinolítica, aumentada resistência periférica à insulina e reduzida performance cardíaca. Os adolescentes que descontinuam o uso de hrGH após terem atingido suas alturas finais também apresentam perfil metabólico desfavorável, como os adultos, porém resta saber se nesta faixa etária os riscos cardiovasculares estão também aumentados. A reinstituição do tratamento com hrGH melhora o perfil lipídico, a massa muscular e a performance cardíaca (já após o primeiro ano de tratamento) e reduz as concentrações de homocisteína, fator de risco independente para complicações cardiovasculares.

\section{USO DE HORMÔNIO DE CRESCIMENTO NA PWS}

Entre as indicações de uso de hrGH aprovadas pela Food and Drug Administration (FDA), algumas indi- cações visam claramente ao problema estatural: deficiência de GH (indicação aprovada em 1985), síndrome de Turner (aprovada em 1996), o pequeno para a idade gestacional (2001) e a baixa estatura idiopática (2003). Por outro lado, o uso de hrGH na insuficiência renal crônica (1993) e na PWS (2000) visam à melhora do perfil metabólico do paciente.

Apesar de se reconhecer que um número importante de pacientes com PWS apresente deficiência de GH, a razão principal do seu uso é proporcionar melhora da relação entre a massa magra e a massa gorda, com restabelecimento da atividade física, da força muscular e da qualidade de vida (19). Por outro lado, como a obesidade é o fator que levará tais pacientes a óbito, deve-se atuar para evitar ou, pelo menos, dificultar o excessivo ganho de peso comumente visto nesta condição clínica. Com o tratamento, também ocorre melhora da massa óssea e do perfil lipídico dos pacientes $(5,20)$.

Em um estudo randomizado de 91 pacientes prépúberes com PWS (42 lactentes, 49 crianças de 3 a 14 anos), houve aumento do SDS de altura de $-2,3$ para $-0,4$ após dois anos de tratamento com hrGH, enquanto em um grupo de crianças não-tratadas não se verificou alteração do SDS de altura. A massa magra aumentou e a porcentagem de gordura corpórea reduziu, apesar de não se normalizar completamente. Por outro lado, na dose de hrGH utilizada $\left(1 \mathrm{mg} / \mathrm{m}^{2} / \mathrm{dia}\right)$, houve aumento de IGF- 1 acima do limite superior da normalidade, o que sugere que se deve monitorar os níveis desse fator de crescimento ao tratar crianças portadoras da PWS com hrGH (21).

Em relação à modificação da composição corpórea e ao consumo energético, Galassetti e cols. realizaram um estudo em 37 pacientes ( 20 do sexo masculino) em idades entre 3 e 38 anos, com vários graus de hipogonadismo, porquanto 20 desses 37 pacientes apresentavam deleção do cromossomo 15 paterno, 17 dissomia uniparental do cromossomo 15 materno e nenhum caso de imprinting. Os pacientes tratados com hrGH apresentaram consumo energético diário de $226 \mathrm{kcal} \mathrm{a}$ mais que o grupo não-tratado (1.296 \pm 78 versus 1.070 $\pm 105 \mathrm{kcal} / \mathrm{dia})(22)$.

No que se refere à idade de início do tratamento com hrGH na PWS, tem sido recomendado início mais precoce, por volta do segundo ano de vida ou mesmo antes, levando-se em consideração os cuidados com a apnéia do sono e a permeabilidade das vias aéreas. Meyers e cols. estudaram um grupo de 25 pacientes com idades entre 4 e 37 meses, randomizados 
para dois anos de terapia com hrGH na dose de $1 \mathrm{mg} /$ $\mathrm{m}^{2} /$ dia ou um ano de observação sem hrGH. Este último grupo, após um ano de observação, era colocado em tratamento com $1,5 \mathrm{mg} / \mathrm{m}^{2} /$ dia por um ano. Os pacientes tratados com hrGH normalizaram seus SDS de altura, tiveram crescimento do perímetro cefálico mais rápido, aumentaram a massa magra e reduziram a massa gorda. Houve, também, melhora cognitiva e da linguagem, comparados aos pacientes que não receberam tratamento no primeiro ano. Apenas um paciente apresentou piora de escoliose e os autores recomendam o uso de hrGH em idade mais precoce na PWS (23).

\section{EFEITOS COLATERAIS DO hrGH NA PWS}

Apesar de os vários efeitos positivos do tratamento de pacientes com PWS com hrGH, deve-se ficar atento para alguns efeitos colaterais. Um deles refere-se à escoliose. Em um estudo de 72 pacientes com escoliose (46 do sexo masculino e 26 do sexo feminino), 41 dos quais em terapia com hrGH e $31 \mathrm{sem}$ hrGH, com idades de l a 49 anos, 33 pacientes $(41,9 \%)$ apresentavam escoliose com ângulo de Cobb > 10 graus. Dos avaliados, $20(48,8 \%)$ entre 41 pacientes que recebiam hrGH e 13 $(41,9 \%)$ de 31 pacientes sem terapia de hrGH tiveram escoliose, não demonstrando diferença estatística. A velocidade de crescimento no primeiro ano de terapia mostrava-se maior no grupo com escoliose do que nos que não a apresentavam $(10,7 \pm 2,54$ versus $8,59 \pm$ 1,92). Entre os 20 pacientes com escoliose que recebiam hrGH, o grau de escoliose progrediu durante a terapia em seis pacientes, melhorou em três e flutuou em um. Muitos pacientes apresentaram progressão da escoliose com a idade, mesmo sem receber hrGH. Os autores concluem que o uso de hrGH aumenta a velocidade de crescimento sem necessariamente levar à escoliose e o início mais precoce do hrGH não parece ser um fator de exacerbação da escoliose (24).

Riedl e cols. publicaram dois casos de óbito em meninas portadoras da PWS após o início de uso de hrGH. Uma das meninas tinha 4,7 anos de idade, apresentou aumento continuado da pressão de artéria pulmonar e faleceu de insuficiência cardiorrespiratória sete semanas após o início de hrGH. A outra menina tinha 9,3 anos de idade, com adicional trissomia do cromossomo 21 e faleceu durante infecção respiratória seis meses após o início de hrGH. Ambas as pacientes estavam acima do peso (peso para altura $127 \%$ e $224 \%$, respectivamente) e suspeita-se que retenção hídrica tenha ocorrido na menina mais jovem. Os autores questionam se os óbitos se deveram ao uso de hrGH ou ao curso natural da doença (25). Outros dois casos de óbito foi descrito por Grugni e cols.: uma menina de 3,9 anos de idade, com evidência laboratorial de deficiência de GH. O tratamento foi iniciado na idade de 3,5 anos, com dose de $0,33 \mathrm{mg} / \mathrm{kg} / \mathrm{semana}$, com a paciente com $130 \%$ a mais do peso ideal para a altura. A hipertrofia de adenóides foi demonstrada previamente e os roncos com apnéia do sono estavam presentes antes do início de hrGH. Tais achados não pioraram durante o tratamento. Quatro meses após o início da terapia com $\mathrm{hrGH}$, a criança foi encontrada morta subitamente pela manhã. O outro caso era de um menino com 6,3 anos de idade, com idade de 6 anos, seu peso era $144 \%$ a mais do seu peso ideal para a altura e apresentava reduzida secreção de $\mathrm{GH}$ aos testes de provocação. Iniciou hrGH na dose de 0,2 $\mathrm{mg} / \mathrm{kg} /$ semana. As dificuldades respiratórias noturnas que o paciente apresentava antes da terapia pioraram após o início do hrGH e notou-se hipertrofia de amígdalas e adenóides. Aos 6,3 anos, faleceu em casa, pela manhã, após crise aguda de apnéia (26).

\section{CONCLUSÕES}

A PWS constitui um desafio diagnóstico e terapêutico. É condição clínica que se inicia com extrema hipotonia, requisitando o concurso de equipe multiprofissional já no início da vida, em que a preocupação maior é com a alimentação, que se torna fator que põe a vida da criança em risco por inanição. Após essa fase, com a melhora da hipotonia, as alterações de apetite e de composição corpórea levam a criança à obesidade extrema, fato que, novamente, coloca a criança e o adolescente em risco de morte. O uso de GH nesta síndrome tem melhorado muito a qualidade de vida dessas crianças e há indicação de início precoce. No entanto, cuidado especial deve ser tomado quanto ao problema da apnéia do sono e da permeabilidade das vias aéreas, de modo que é obrigatório a polissonografia, cuidadoso exame otorrinolaringológico prévio à instituição da terapia e cuidadoso monitoramento durante o tratamento. Muitas dessas crianças apresentam, de fato, deficiência de $\mathrm{GH}$, de modo que a melhora do padrão estatural também pode ser esperada. 


\section{REFERÊNCIAS}

1. Prader A, Labhart A, Willi H. Ein syndrom von adipositas, kleinwuchs, kryptorchismus und oligophernie nach myotonieartigem zustand im neugeborenenalter. Schweiz Med Wochenschr. 1956;86:1260-1.

2. Gillessen-Kaesbach G, Gross S, Kaya-Westerloh S, Passarge E, Horsthemke B. DNA methylation based testing of 450 patients suspected of having Prader-Willi syndrome. J Med Genet. 1995;32:88-92.

3. Holm V, Cassidy SB, Butler MG, Hanchett JM, Greenswag LR, Whitmann BYGF. Prader-Willi syndrome: consensus diagnostic criteria. Pediatrics. 1993;91:398-402.

4. Stafler P, Wallis C, Grugni G, Crinò A, Bosio L, Corrias A, et al. The Italian national survey for Prader-Willi syndrome: an epidemiologic study. Am J Med Genet. 2008 Apr 1;146(7):861-72.

5. Kuo JY, Dichtchekenian V, Manna TD, Kuperman H, Damiani D, Setian N. Síndrome de Prader-Willi: aspectos metabólicos associados ao tratamento com hormônio de crescimento. Arq Bras Endocrinol Metab. 2007;51(1):92-8.

6. Crinò A, DiGiorgio G, Schiaffini R, Fierabracci A, Spera S, Maggioni $A$, et al. Central precocious puberty and growth hormone deficiency in a boy with Prader-Willi syndrome. Eur J Pediatr. 2008 Feb 27 (Epub ahead of print).

7. Lindgren AC. Somatropin therapy for children with PraderWilli syndrome: guidelines for use. Treat Endocrinol. 2006;5:223-8.

8. Krochik AG, Ozuna B, Torrado M, Chertkoff L, Mazza C. Characterization of alterations in carbohydrate metabolism in children with Prader-Willi syndrome. J Pediatr Endocrinol Metab. 2006;19:911-8.

9. Festen DA, van Toorenenbergen A, Duivenvoorden HJ, HokkenKoelega AC. Adiponectin levels in prepubertal children with Prader-Willi syndrome before and during growth hormone therapy. J Clin Endocrinol Metab. 2007;92(4):1549-54.

10. Allen DB, Carrel AL. Growth hormone therapy for Prader-Willi syndrome: a critical appraisal. J Pediatr Endocrinol Metab. 2004;17:1297-306.

11. Carrel AL, Allen DB. Prader-Willi syndrome: how does growth hormone affect body composition and physical function? J Pediatr Endocrinol Metab. 2001;14:1445-51.

12. Butler MG, Meaney FJ. Standards for selected anthropometric measurements in Prader-Willi syndrome. Pediatrics. 1991; 88:853-60.

13. Cassidy SB. Prader-Willi syndrome. J Med Genet. 1997;34: 917-23.

14. Nam SY, Mardcus C. Growth hormone and adipocyte function in obesity. Horm Res. 2000;53 Suppl 1:S87-97.

15. Nonogaki K. Ghrelin and feedback systems. Bitam Horm. 2008;77:149-70.

16. Lanes R, Soros A, Gunczler P, Paoli M, Carrillo E, Villaroel O, et al. Growth hormone deficiency, low Iwvels of adiponectin, and unfavorable plasma lipid and lipoproteins. J Pediatr. 2006;149:324-9.
17. Johannsson G, Albertsson-Wikland K, Bengtsson BA. Discontinuation of growth hormone $(\mathrm{GH})$ treatment: metabolic effects in GH-deficient and $\mathrm{GH}$-sufficient adolescent patients compared with control subjects. Swedish Study Group for Growth Hormone Treatment in Children. J Clin Endocrinol Metab. 1999;84:4516-24.

18. Salerno M, Esposito V, Farina V, Radetti G, Umbaldo A, Capalbo $D$, et al. Improvement of cardiac performance and cardiovascular risk factors in children with $\mathrm{GH}$ deficiency after two years of $\mathrm{GH}$ replacement therapy: an observational, open, prospective, case-control study. J Clin Endocrinol Metab. 2006;91:288-95.

19. Bertella L, Mori I, Grugni G, Pignatti R, Ceriani F, Molinari E, et al. Quality of life and psychological well-being in GH-treated, adult PWS patients: a longitudinal study. J Intellect Disabil Res. 2007;51:302-11.

20. Stafler $P$, Wallis C. Prader-Willi syndrome: who can have growth hormone? Arch Dis Child. 2008;93(4):341-5.

21. Festen DA, de Lind van Wijngaarden $R$, van Eekelen M, Otten BJ, Wit JM, Duivenvoorden HJ, et al. Randomized controlled growth hormone trial: effects on anthropometry, body composition, and body proportion in a large group of children with Prader-Willi syndrome. Clin Endocrinol (Oxf). 2008 Mar 18 (Epub ahead of print).

22. Galassetti P, Saetrum Opgaard O, Cassidy SB, Pontello A. Nutrient intake and body composition variables in Prader-Willi syndrome - effect of growth hormone supplementation and genetic subtype. J Pediatr Endocrinol Metab. 2007;20:491-500.

23. Meyers SE, Whitman BY, Carrel AL, Moerchen V, Bekx MT, Allen DB. Two years of growth hormone therapy in young children with Prader-Willi syndrome: physical and neurodevelopmental benefits. Am J Med Genet. 2007;143:443-8.

24. Nagai T, Obata K, Ogata T, Murakami N, Katada Y, Yoshino A, et al. Growth hormone therapy and scoliosis in patients with Prader-Willi syndrome. Am J Med Genet. 2006;140:1623-7.

25. Riedl S, Blümel P, Zwiauer K, Frisch H. Death in two female Prader-Willi syndrome patients during the early phase of growth hormone treatment. Acta Paediatr. 2005;94:974-7.

26. Grugni G, Livieri C, Corrias A, Sartorio A, Crinò A. Genetic Obesity Study Group of the Italian Society of Pediatric Endocrinology and Diabetology. Death during GH therapy in children with Prader-Willi syndrome: description of two new cases. J Endocrinol Invest. 2005;28:554-7.

Endereço para correspondência:

Durval Damiani

Rua Bela Cintra, 2117, apto. 9

01415-002 São Paulo, SP

E-mail: durvald@iconet.com.br 